\title{
Cardiac Troponin I Levels in Hospitalized COVID- 19 Patients as a Predictor of Severity and Outcome: A Retrospective Cohort Study
}

Jabar Ali ${ }^{1}$, Fahad R. Khan ${ }^{2}$, Rizwan Ullah ${ }^{2}$, Zair Hassan ${ }^{2}$, Safi Khattak ${ }^{3}$, Gul Lakhta ${ }^{4}$, Nooh Zad Gul ${ }^{2}$, Rahman Ullah ${ }^{2}$

1. Cardiology/Interventional Cardiology, Lady Reading Hospital, Peshawar, PAK 2. Cardiology, Lady Reading Hospital, Peshawar, PAK 3. Cardiology, Lady Reading Hospital, Peshwar, PAK 4. Gynecology and Obstetrics, Lady Reading Hospital, Peshawar, PAK

Corresponding author: Fahad R. Khan, fahadraja78@gmail.com

\section{Abstract \\ Introduction}

The COVID-19 (coronavirus disease) has affected millions of people, wreaking havoc worldwide. World Health Organization (WHO) labelled this disease as a serious threat to public health since its rapid spread from Wuhan, China. The respiratory manifestations of COVID-19 are common, but myocardium involvement causing myocardial injury and rise in cardiac markers is much less discussed.

\section{Materials and methods}

We conducted this retrospective cohort study from 1st April 2020 to 1st October 2020. Data was collected from the Hospital Management and Information System (HMIS) based on inclusion criteria. We used the Cox proportional hazard regression model for survival analysis, estimated the probability curves of survival using the Kaplan-Meier method, and contrasted it with the log-rank test.

\section{Results}

Among the 466 patients, 280 (69\%) were male; the rest were female. The majority were both hypertensive and diabetic, and one-third had a myocardial injury on arrival. The most frequent symptoms in more than half of the patients (51.90\%) included a combination of fever, dry cough, and shortness of breath. Out of 466 patients, 266 patients were discharged, and 200 did not survive. In our study, 168 (36.05\%) patients had a cardiac injury; among them, 38 (22.61\%) were in the discharge group, and the remaining 130 (77.39\%) patients were in the nonsurvivor group. Our study results showed that the mortality rate was higher in patients with high cardiac troponin I (cTnI) levels (hazard ratio [HR] 3.61) on admission.

\section{Conclusion}

Review began 03/13/2021 Review ended 03/22/2021 Published 03/23/2021

\section{() Copyright 2021}

Ali et al. This is an open access article distributed under the terms of the Creative Commons Attribution License CC-BY 4.0., which permits unrestricted use, distribution, and reproduction in any medium, provided the original author and source are credited.
Our result concluded that measuring cTnI levels on presentation could help predict the severity and outcome in COVID-19 patients. It will allow physicians to triage patients and decrease mortality.

Categories: Cardiology, Internal Medicine, Infectious Disease

Keywords: covid-19 pandemic, covid-19 virus disease, sars-cov-2 infection, covid-19 outbreak, covid 19, cardiac troponin i, troponin i, troponin-i and d-dimers, crp levels

\section{Introduction}

The pandemic of COVID-19 (coronavirus disease) is the major public health concern of the century. For several decades to come, this outbreak's health, financial, and cultural consequences will be experienced by human beings [1]. The virus that caused this disease outbreak in 2019, known as SARS-CoV-2 (severe acute respiratory syndrome coronavirus-2), is much more infectious than severe acute respiratory syndrome (SARS) and Middle East respiratory syndrome coronavirus (MERS-CoV) [2]. Besides its impact on the lungs, the novel coronavirus can invade several vital organs (kidneys, brain, and heart), trigger cytokine storm (acute hyperinflammatory response associated with the release of various inflammatory cytokines), followed by multiple organ failure. Of these organs, the heart is a critical target [3]. A literature search showed viral myocarditis and myocardial damage as perpetrators and one of the leading causes of death by COVID-19[3].

There is ample evidence of COVID-19-related rise in cardiac troponin T and cardiac troponin I (cTnI) beyond the 99th percentile reference point in the literature [4]. The severe cardiac injury is defined as when the cTnI serum concentration exceeds the upper reference point $(0.4 \mathrm{ng} / \mathrm{ml})$ three times [5]. Studies have shown that COVID-19 patients with raised cTnI levels had poor prognosis. The risk of ventilation support and intensive care unit need rises by up to five times [6]. Literature is available but scarce on this topic. The 
current study is the first retrospective cohort study from Pakistan conducted on the COVID-19 infected patients. It established a relationship between cTnI levels and disease severity and outcome and validated findings of earlier studies. It will set a path for other researchers to follow suit to understand better the predictive value of cardiac biomarkers and myocardial damage in COVID-19.

\section{Materials And Methods \\ Study Population}

We conducted this study on all confirmed consecutive COVID-19 patients presented to our hospital between 1st April 2020 to 1st October 2020. We labelled patients as COVID-19 according to interim guidelines from the World Health Organization. This study proceeded after approval from the Lady Reading Hospital's Ethical Review Board (ERB/REF\#585).

\section{Data collection}

We retrieved the retrospective records of 466 patients from the hospital management information system (HMIS). After informed consent from patients, we included them based on the inclusion and exclusion criteria. Variables collected were demographics, presenting complaints, comorbidities, laboratory investigations, patients' outcome (death or discharge from hospital), and time to the event. The reference level for normal cTnI level in our hospital is less than $0.4 \mathrm{ng} / \mathrm{ml}$. Among the laboratory investigations were the baseline investigations (white blood cells [WBC]), acute phase reactants (serum ferritin, C-reactive protein [CRP], and D-dimers), and cardiac troponin I level (cTnI).

\section{Study participants}

Our study's inclusion criteria included patients who had a positive real-time polymerase chain reaction (RTPCR) test and were older than 18 years of age. Patients presenting with acute decompensated heart failure (ADHF), hypertensive crisis, and cardiovascular accidents (CVA) were not included in the study. These can cause a pseudo rise in CTnI. COVID-19 patients with a history of coronary revascularization either through percutaneous intervention (PCI) or coronary artery bypass graft (CABG) surgery were not enrolled in the study. Patients with severe valvular disease, rhythm disorders such as tachyarrhythmia or bradyarrhythmia, implanted pacemaker, chronic obstructed lung disease (COPD), chronic kidney disease (CKD), along with those who did not undergo cTnI testing at admission were excluded.

\section{Statistical analysis}

We performed descriptive analyses after stratifying cTnI levels into normal (0.00-0.4 ng/ml) and elevated levels, i.e., $>0.4 \mathrm{ng} / \mathrm{ml}$ ). We then presented continuous variables as mean and standard deviation and categorical variables as frequency rates and percentages. After that, we applied the Mann-Whitney U ranksum test for testing the mean values of continuous variables and fisher's exact test for categorical variables. We then applied multivariate regression using the Cox Proportional Hazards Model to determine the independent risk factors of mortality during hospitalization. We used this model with and without adjusting for other variables. These variables included demographics (age, sex), underlying comorbidities, clinical variable (Body Mass Index [BMI]), and laboratory measurements (serum D-dimers, serum ferritin, cTnI levels, WBCs, and CRP). After that, we estimated the probability curves of survival using the KaplanMeier method and contrasted them with the log-rank test.

\section{Results}

During the study period from 1st April till 1st October 2020, we included 466 patients. The patients' median age (SD) was $55.01 \pm 13.49$ years (range $22-90$ years), and the mean BMI was $24.94 \pm 4.81$. Among these patients, $280(60.09 \%)$ were male, and 186 (39.91\%) were female. The most common comorbidities were diabetes mellitus and hypertension $(n=80,17.17 \%)$. The most common symptoms were dry cough, fever, and shortness of breath $(n=242,51.93 \%)$. A total of $266(57.08 \%)$ survived, and we discharged them, while 200 (42.92\%) died. cTnI was raised in 168 (36.1\%) patients. 38 (22.61\%) were in the survivor group, whereas 130 (77.39\%) were in the nonsurvivor group. Besides cTnI, we measured WBCs and acute phase reactants such as serum ferritin, CRP, and serum D-dimer levels in every patient. These baseline line demographics, clinical characteristics, and the patients' investigations are given in Table 1 . 


\section{Cureus}

Variable

Gender

Male

Female

Symptoms

Dry Cough

Fever

Shortness of breath

Dry cough, fever, and shortness of breath

\section{Cardiac Troponin I (cTnl) levels}

Normal cTnl level

High cTnl level

Serum D-dimer

Normal

More $>400$

more $>1000$

Comorbidities

DM

HTN

DM and HTN

IHD

No Comorbidities

Serum Ferritin

Normal

High (more than 200)

very high (more than 1000)

C-reactive proteins (CRP)

Normal

High

Outcome

Discharge

Death

TABLE 1: Baseline demographic and clinical characteristics of the patients

n-frequency; \%-percentage; DM-diabetes; HTN-hypertension; IHD-ischemic heart disease; cTnl-cardiac troponin I; CRP-C-reactive proteins

A two-tailed Mann-Whitney two-sample rank-sum test was conducted to examine significant differences in age and BMI among patients who died and those who were discharged. The result of the Mann-Whitney U test was not significant for age $(\mathrm{p}=.198)$, whereas significant for BMI $(\mathrm{p}<.001)$. This suggested that the BMI distribution for the discharge category differed significantly from the BMI distribution for the death category. We then compared categorical variables among groups using the fisher's exact test. 


\section{Cureus}

\section{Unadjusted Cox proportional hazards model}

An unadjusted Cox proportional hazards model was conducted to determine whether cTnI, on admission, had a significant effect on the hazard outcome without adjusting for other variables. The outcome discharge category was used to show survival, while the death category represented a hazard event. The coefficient for the unadjusted high category of cTnI was significant; $\mathrm{HR}=6.09, \mathrm{p}<.001$, indicating that observation in the high cTnI category will have a hazard, i.e., 6.09 times as large as the normal cTnI levels category shown in Table 2 .

\begin{tabular}{|c|c|c|c|c|c|c|}
\hline Variable & B & SE & $95 \% \mathrm{Cl}$ & $\mathbf{z}$ & $\mathbf{p}$ & HR \\
\hline High troponin I & 1.81 & 0.16 & {$[1.50,2.11]$} & 11.62 & $<.001$ & 6.09 \\
\hline
\end{tabular}

\section{TABLE 2: Unadjusted Cox proportional hazards regression coefficients for troponin I}

Cl-confidence interval; HR-hazard ratio; B-unstandardized beta; SE-standard error; z-ratio of regression coefficient to its standard error

\section{Adjusted Cox proportional hazards model}

We then applied the Cox proportional hazards model adjusting for demographics (age, sex), underlying comorbidities, clinical variable (body mass index), and laboratory measurements (serum D-dimers, serum ferritin, cTnI, WBCs, and C-reactive protein). The outcome discharge category was used to show survival, while the death category represented a hazard event. The Cox proportional hazards model showed an increased risk of death with high cTnI levels at admission. Patients with hypertension and diabetes together, history of ischemic heart disease, raised CRP, and D-dimer levels increased the risk of dying. While age, BMI, gender, hemoglobin (HB), serum ferritin, and WBCs did not affect the outcome (Table 3). We included a Kaplan-Meier survival probability plot for adjusted cTnI and comorbidities. Each plot represented the survival probabilities over time (Figures 1,2). 


\section{Cureus}

\begin{tabular}{|c|c|c|c|c|c|c|}
\hline Variable & B & SE & $95 \% \mathrm{Cl}$ & $\mathbf{z}$ & p & HR \\
\hline Age & 0.01 & 0.01 & {$[-0.01,0.02]$} & 0.88 & . 379 & 1.01 \\
\hline BMI & 0.02 & 0.02 & {$[-0.02,0.05]$} & 0.88 & .377 & 1.02 \\
\hline Gender (Female) & -0.01 & 0.15 & {$[-0.31,0.28]$} & -0.10 & .923 & 0.99 \\
\hline Hypertension & 0.01 & 0.22 & {$[-0.42,0.44]$} & 0.05 & .964 & 1.01 \\
\hline Diabetes Mellitus and Hypertension & 1.42 & 0.28 & {$[0.88,1.96]$} & 5.14 & $<.001$ & 4.14 \\
\hline Ischemic heart disease & 0.62 & 0.22 & {$[0.18,1.05]$} & 2.78 & . 005 & 1.85 \\
\hline No Comorbidities & -1.46 & 0.42 & {$[-2.28,-0.64]$} & -3.47 & $<.001$ & 0.23 \\
\hline WBC Mildly Raised (B/w 11000 and 15000) & 0.22 & 0.20 & {$[-0.18,0.62]$} & 1.08 & 279 & 1.25 \\
\hline WBCs moderately raised (between 15000 and 20000) & 0.23 & 0.22 & {$[-0.21,0.67]$} & 1.02 & .308 & 1.26 \\
\hline WBCs severely raised (more than 20,000 ) & 0.57 & 0.21 & {$[0.16,0.98]$} & 2.73 & . 006 & 1.77 \\
\hline CRP & 0.87 & 0.31 & {$[0.27,1.48]$} & 2.84 & . 005 & 2.39 \\
\hline Ferritin high (more than 200) & -0.12 & 0.32 & {$[-0.75,0.51]$} & -0.36 & .716 & 0.89 \\
\hline Ferritin very high (more than 1000) & -0.52 & 0.29 & {$[-1.10,0.05]$} & -1.79 & . 074 & 0.59 \\
\hline High troponin I (cTnl) & 1.28 & 0.30 & {$[0.70,1.86]$} & 4.33 & $<.001$ & 3.61 \\
\hline D-dimer high (more than 400) & 1.02 & 0.42 & {$[0.19,1.85]$} & 2.40 & .016 & 2.76 \\
\hline D-dimer very high (more than 1000) & 1.55 & 0.38 & {$[0.81,2.29]$} & 4.11 & $<.001$ & 4.70 \\
\hline
\end{tabular}

TABLE 3: Cox proportional hazards regression model for age, BMI, gender, comorbidities, WBCs, CRP, ferritin, cardiac troponin I (cTnl), and D-dimers

P<0.05; DM-diabetes mellitus; HTN-hypertension; WBCs-white blood cells; CRP-C-reactive proteins; BMI-body mass index; CI-confidence interval; HR-hazard ratio; B-unstandardized beta; SE-standard error; z-ratio of regression coefficient to its standard error

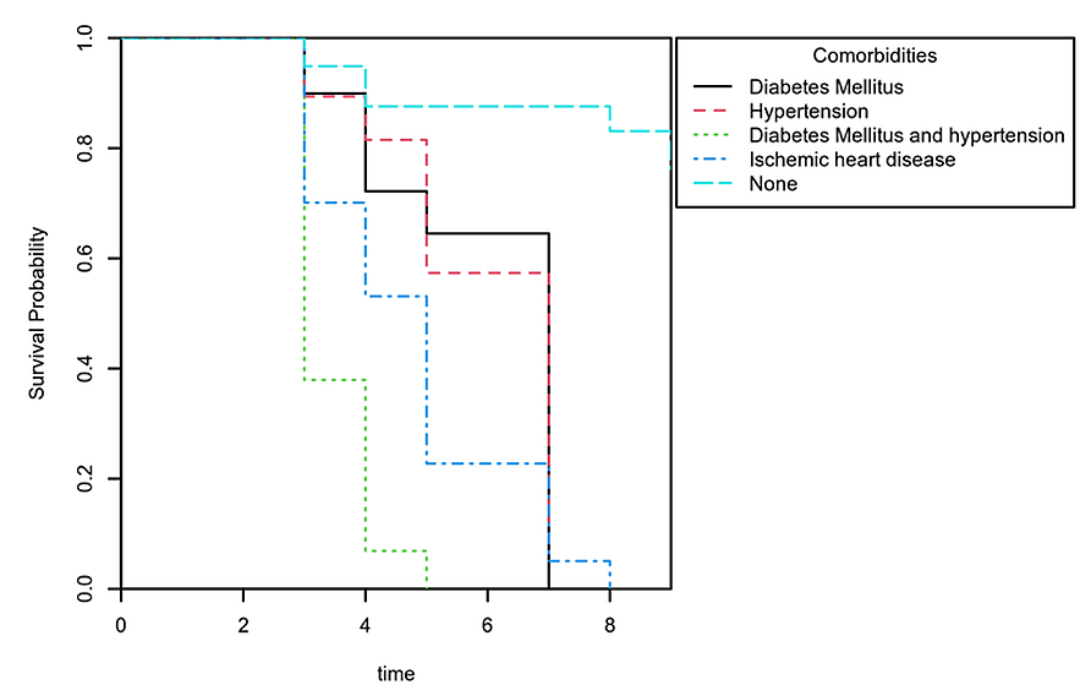

FIGURE 1: Kaplan-Meier survival plot of outcome grouped by comorbidities 


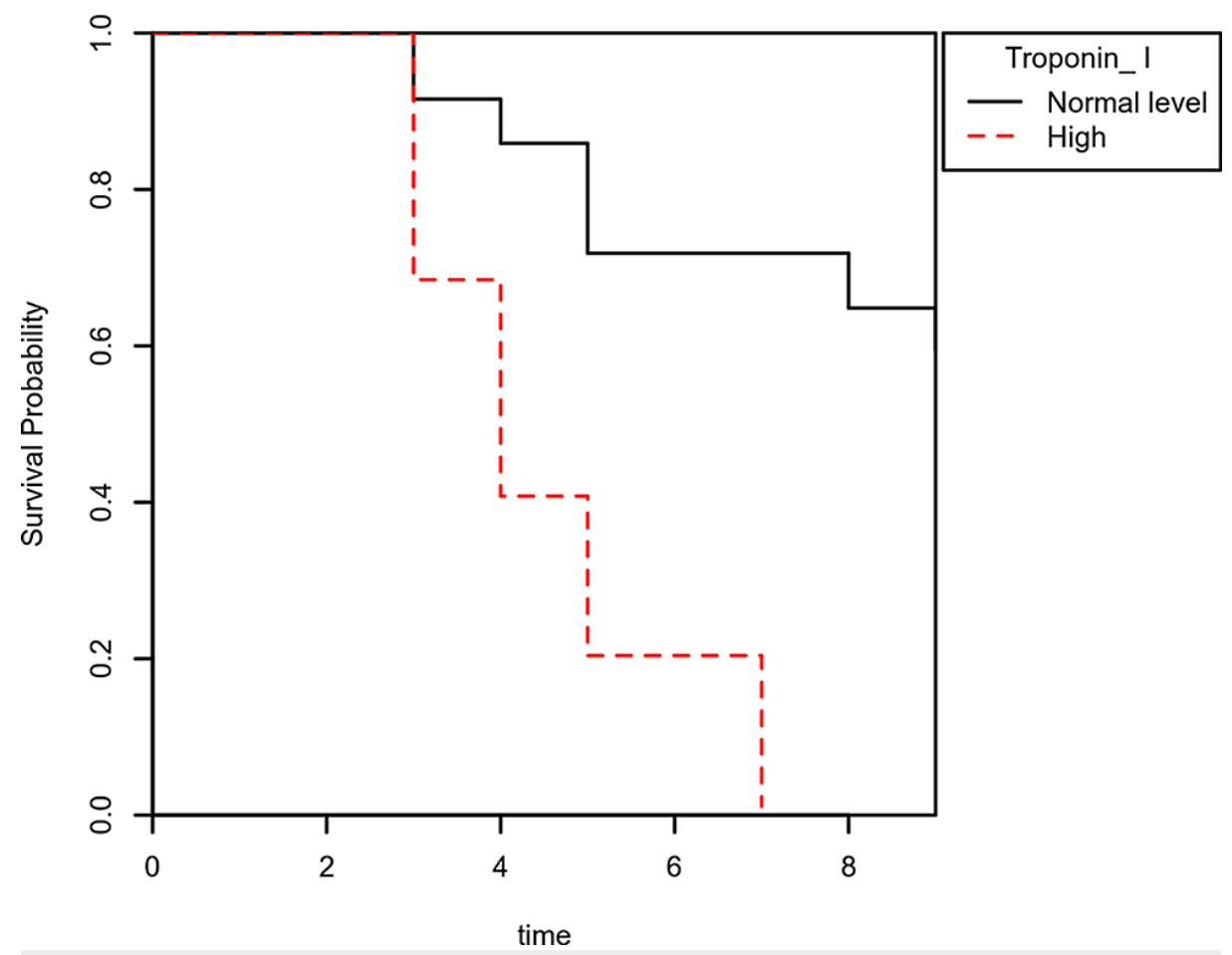

FIGURE 2: Kaplan-Meier survival plot of outcome grouped by cardiac troponin I (cTnl) levels

\section{Discussion}

The present study established a significant association between cardiac injury (detected by measuring cTnI) and outcome in hospitalized COVID-19 patients. To the best of our knowledge, this is the first and most extensive study to date in Pakistan investigating the impact of cardiac biomarkers on outcome in hospitalized COVID-19 patients. Our findings emphasized the inclusion of cardiac troponin I levels into everyday practice to detect, predict the severity of, and manage COVID-19 virus-infected patients. It has been shown to contribute to the COVID-19-related cardiac injury [3-6]. Male and elderly patients formed the bulk of our study group. The mean age $(55.01 \pm 13.49$ years $)$ of our research population was less than the mean age of patients in previous researches [6-8]. This trend showed a propensity of COVID-19 virus disease in young patients in this part of the world. The mean BMI was $(24.94 \pm 4.81)$, which was lower than the BMI of patients in previous studies [7-9]. The lower BMI suggested that patients in Pakistan infected by the SARS-CoV-2 virus were leaner than those in other parts of the world. Many studies have shown the rising mortality with higher BMI values [7-11]. While on the contrary, our research's Cox regression analysis revealed that BMI did not affect the outcome (HR 1.02 [confidence interval (CI) 95\%, -0.02,0.05], p=.377).

In our study, mortality was higher in patients with high cTnI levels (HR 3.61 [CI 95\%, 0.70,1.86], p<.001), patients suffering from both hypertension and diabetes together (HR 4.14 [CI 95\%, 0.88,1.96], p<.001), and in patients who had a history of ischemic heart disease (IHD) (HR 1.85 [CI 95\%, 0.18,1.05], p=.005). These findings are in line with the findings of Santos et al. [12].

Viral diseases, such as influenza, can often present as cardiac injury. Several studies have endorsed this statement. In the $2009 \mathrm{H} 1 \mathrm{~N} 1$ pandemic, retrospective findings showed that $54 \%$ of patients had cardiac insult [13]. Cases of myocarditis were recorded in 2012 by MERS-CoV [13-15]. Likewise, coronavirus disease (COVID-19) is a lethal infectious disease that has infected over 58.8 million people worldwide as of November 2020. It surfaced in Wuhan, China and has spread, resulting in a growing pandemic [16-18]. Given the rising incidence of COVID-19 virus disease, research in the domain was much needed. The rapid surge in COVID-19 demanded urgent attention both in the diagnostic and therapeutic contexts. This urgency led us to conduct this research on admitted patients of COVID-19 virus disease.

Multiple body organs are infected by COVID-19 virus disease [19]. Among these, cardiac involvement is not rare. Cardiac involvement has also been reflected in our research: 168 (36.05\%) patients have raised levels of cTnI. More than half of the patients in our sample died from a myocardial injury, consistent with the results of Yange et al., Pzizzini et al., and Gao et al. [17,19,20]. In contrast, 19\% died with COVID-19 infection with raised cTnI level in a study by Shi et al. [21]. Our research included more patients with myocardial injury and elevated cTnI levels than previously published studies [16,22-26]. As evident in previous studies, the rate and quasi nature of abnormal cTnI levels in COVID-19-infected cases prompted 
clinicians to measure cTnI levels only when they suspected acute myocardial infarction. In comparison, we have tried to determine that all patients infected with COVID-19 should be screened for myocardial injury by carrying out cTnI levels. Our findings demonstrated that cTnI acted as a marker of severity in COVID-19 virus disease and was raised in around one-third of COVID-19 patients.

Multiple studies have examined the relationship between rising cardiac enzymes and poor prognosis in COVID-19 virus disease. These findings were in line with our research. The coefficient for unadjusted elevated cTnI levels was significant (HR 6.09 [CI 95\%, 1.50,2.11], p<.001), showing that a rise in cTnI has a hazard that is 6.09 times as large as normal cTnI levels.

According to Huang et al., diabetes mellitus has increased mortality and disease severity in COVID-19 [27]. In our study, people with only diabetes as a sole risk factor did not have high mortality, while those with both diabetes and hypertension as their risk factors were at increase risk of dying (HR 4.14 [CI 95\%, $0.88,1.96], \mathrm{p}<.001)$.

In a study conducted by Nie et al., a total of 311 patients diagnosed with COVID-19 virus disease were enrolled. Among them, 111 patients died, and 200 survived. The cardiac injury was present in 103 (33.1\%) patients. 12 of these patients were in the discharged category, and 91 were in the nonsurvivor category. Multivariable logistic regression was carried out, which declared cardiac troponin I as a predictor of mortality in COVID-19 patients [28]. In comparison, we conducted our study on 466 hospitalized patients, of which 200 died and 266 survived. cTnI was raised in 168 patients. 38 of them were in the discharge group, while 130 were in the nonsurvivor category. We conducted the Cox proportional hazards model, which showed an increased risk of dying in patients with high cTnI levels at admission, which is consistent with the findings of Nie et al. We did not go into details of the pathogenesis involved in each case. We only aimed to establish the fact that there is an association between cardiac markers, i.e., cTnI and disease severity in COVID-19 affected patients.

\section{Limitations}

Our study had certain limitations; First, our study was a single-centred study. Second, we did not consider the underlying pathology of myocardial injury leading to a rise in cTnI levels. Third, we did not measure serial (cTnI) levels because of limited resources, which might have included or excluded patients. Our fourth limitation was that we did not consider all clinical and pathological confounders owing to the excessive patient burden, limited resources, and the risk of getting infected. We did not follow these patients after discharge from the hospital.

\section{Conclusions}

The infection of COVID-19 is growing vigorously. With the rising number of new cases, awareness regarding its clinical presentations and diagnosis become more comprehensive. Cardiac biomarkers are typically elevated in patients with COVID-19 disease. The rise in cTnI can be explained by several mechanisms, from myocarditis to cytokine activity, inflicting myocardial injury. These mechanisms are controversial, and none has proved to be the main cause behind raised cTnI levels. Our study did not focus on the pathophysiology and concluded that hospitalized patients diagnosed with COVID-19 and raised cTnI are at increased risk of dying. In other words, high cTnI levels is an indicator of the severity and poorer outcome in patients with COVID-19 virus disease. Measuring cTnI levels in COVID-19 virus disease can direct physicians to triage patients and decrease mortality. Research on a larger scale is needed to drive a global reaction against COVID-19. Cardiac troponin elevations can be considered as a crude marker of severity. This has been illustrated in our study.

\section{Additional Information}

\section{Disclosures}

Human subjects: Consent was obtained or waived by all participants in this study. Ethical Review Board issued approval REF\#676. This is to certify that Ethical Approval has been granted to Dr Jabar Ali, Dr Fahad Raja Khan, Dr Rizwan Ullah, Dr Zair Hassan, Dr Safi Ullah Khattak, Dr Gul Lakhta, Dr Nooh Zaad Gul and Dr Rahman Ullah, MTI/LRH, Peshawar, for the study titled "Cardiac Troponin-I (cTnI) levels in Hospitalized COVID-19 Patients as a predictor of severity and Outcome: A Retrospective Cohort Study". Animal subjects: All authors have confirmed that this study did not involve animal subjects or tissue. Conflicts of interest: In compliance with the ICMJE uniform disclosure form, all authors declare the following: Payment/services info: All authors have declared that no financial support was received from any organization for the submitted work. Financial relationships: All authors have declared that they have no financial relationships at present or within the previous three years with any organizations that might have an interest in the submitted work. Other relationships: All authors have declared that there are no other relationships or activities that could appear to have influenced the submitted work.

\section{References}

1. Boukhris M, Hillani A, Moroni F, et al.: Cardiovascular implications of the COVID-19 pandemic: a global 
perspective. Can J Cardiol. 2020, 36:1068-1080. 10.1016/j.cjca.2020.05.018

2. Wu Y, Ho W, Huang Y, et al.: SARS-CoV-2 is an appropriate name for the new coronavirus . Lancet. 2020, 395:949-950. 10.1016/S0140-6736(20)30557-2

3. Deng Q, Hu B, Zhang Y, et al.: Suspected myocardial injury in patients with COVID- 19: evidence from front-line clinical observation in Wuhan, China. Int J Cardiol. 2020, 311:116-121. 10.1016/j.ijcard.2020.03.087

4. Gaze DC: Clinical utility of cardiac troponin measurement in COVID-19 infection . Ann Clin Biochem. 2020, 57:202-205. 10.1177/0004563220921888

5. Chilimuri S, Sun H, Aleman A, et al.: Predictors of mortality in adults admitted with COVID-19: retrospective cohort study from New York City. West J Emerg Med. 2020, 21:779. 10.5811/westjem.2020.6.47919

6. Pan F, Yang L, Li Y, et al.: Factors associated with death outcome in patients with severe coronavirus disease-19 (COVID-19). Int J Cardiol. 2020, 7:1281-1292. 10.7150\%2Fijms.46614

7. Lombardi CM, Carubelli V, Iorio A, et al.: Association of troponin levels with mortality in Italian patients hospitalized with coronavirus disease 2019: results of a multicenter study. JAMA Cardiol. 2020, 5:1274-1280. 10.1001/jamacardio.2020.3538

8. Raad M, Dabbagh M, Gorgis S, et al.: Cardiac injury patterns and inpatient outcomes among patients admitted with COVID-19. Am J Cardiol. 2020, 133:154-161. 10.1016/j.amjcard.2020.07.040

9. Suleyman G, Fadel RA, Malette KM, et al.: Clinical characteristics and morbidity associated with coronavirus disease. JAMA Netw Open. 2020, 3:e2012270. 10.1001/jamanetworkopen.2020.12270

10. Lala A, Johnson KW, Januzzi JL, et al.: Prevalence and impact of myocardial injury in patients hospitalized with COVID-19 infection. J Am Coll Cardiol. 2020, 76:533-546. 10.1016/j.jacc.2020.06.007

11. Ribes A, Vardon-Bones F, Mémier V, et al.: Thromboembolic events and Covid-19. Adv Biol Regul. 2020, $77: 100735.10 .1016 /$ j.jbior.2020.100735

12. Santos CS, Morales CM, Álvarez ED, Castro CÁ, Robles AL, Sandoval TP: Determinants of COVID-19 disease severity in patients with underlying rheumatic disease. Clin Rheumatol. 2020, 39:2789-2796. 10.1007/s10067-020-05301-2

13. Hendrickson CM, Matthay MA: Viral pathogens and acute lung injury: investigations inspired by the SARS epidemic and the 2009 H1N1 influenza pandemic. Semin Respir Crit Care Med. 2013, 34:475-486. 10.1055/s0033-1351122

14. Alhogbani T: Acute myocarditis associated with novel Middle East respiratory syndrome coronavirus . Ann Saudi Med. 2016, 36:78-80. 10.5144/0256-4947.2016.78

15. Huang C, Wang Y, Li X, et al.: Clinical features of patients infected with 2019 novel coronavirus in Wuhan, China. Lancet. 2020, 395:497-506. 10.1016/S0140-6736(20)30183-5

16. Richardson S, Hirsch JS, Narasimhan M, et al.: Presenting characteristics, comorbidities, and outcomes among 5700 patients hospitalized with COVID-19 in the New York City area. JAMA. 2020, 323:2052-2059. 10.1001/jama.2020.6775

17. Yang X, Yu Y, Xu J, et al.: Clinical course and outcomes of critically ill patients with SARS-CoV-2 pneumonia in Wuhan, China: a single-centre, retrospective, observational study. Lancet Respir Med. 2020, 8:475-481. 10.1016/S2213-2600(20)30079-5

18. Fisher HK: Hypoxemia in COVID-19 patients: An hypothesis . Medical Hypotheses. 2020, 143:110022. 10.1016/i.mehy.2020.110022

19. Pizzini A, Burkert F, Theurl I, Weiss G, Bellmann-Weiler RJH: Prognostic impact of high sensitive Troponin T in patients with influenza virus infection: a retrospective analysis. Heart Lung. 2020, 49:105-109. 10.1016/j.hrtlng.2019.05.009

20. Gao C, Wang Y, Gu X, et al.: Association between cardiac injury and mortality in hospitalized patients infected with avian influenza A (H7N9) virus. Crit Care Med. 2020, 48:451-458. 10.1097/CCM.0000000000004207

21. Shi S, Qin M, Shen B, et al.: Association of cardiac injury with mortality in hospitalized patients with COVID-19 in Wuhan, China. JAMA Cardiol. 2020, 5:802-810. 10.1001/jamacardio.2020.0950

22. Zhou F, Yu T, Du R, et al.: Clinical course and risk factors for mortality of adult inpatients with COVID-19 in Wuhan, China: a retrospective cohort study. Lancet. 2020, 395:1054-1062. 10.1016/s0140-6736(20)305663

23. Wang D, Hu B, Hu C, et al.: Clinical characteristics of 138 hospitalized patients with 2019 novel coronavirus-infected pneumonia in Wuhan, China. JAMA. 2020, 323:1061-1069. 10.1001/jama.2020.1585

24. Guo T, Fan Y, Chen M, et al.: Cardiovascular implications of fatal outcomes of patients with coronavirus disease 2019 (COVID-19). JAMA Cardiol. 2020, 5:811-818. 10.1001/jamacardio.2020.1017

25. Wei JF, Huang FY, Xiong TY, et al.: Acute myocardial injury is common in patients with COVID-19 and impairs their prognosis. Heart. 2020, 106:1122-1124. 10.1136/heartjnl-2020-317007

26. Xu XW, Wu XX, Jiang XG, et al.: Clinical findings in a group of patients infected with the 2019 novel coronavirus (SARS-Cov-2) outside of Wuhan, China: retrospective case series. BMJ. 2020, 368:m606. 10.1136/bmj.m606

27. Huang I, Lim MA, Pranata R: Diabetes mellitus is associated with increased mortality and severity of disease in COVID-19 pneumonia-a systematic review, meta-analysis, and meta-regression. Diabetes Metab Syndr. 2020, 14:395-403. 10.1016/j.dsx.2020.04.018

28. Nie SF, Yu M, Xie T, et al.: Cardiac troponin I is an independent predictor for mortality in hospitalized patients with COVID-19. Circulation. 2020, 142:608-610. 10.1161/CIRCULATIONAHA.120.048789 